\title{
Jean Baudrillard und das Verschwinden Europas
}

Roland Barthes hat in seinem gesamten Oeuvre einen langen und durchaus widerspruchsvollen Weg zurückgelegt, der ihn ebenso als Strukturalisten wie danach als Poststrukturalisten und Postmodernen bekannt machte. Seine Arbeit an und mit den Formen des Reiseberichts zeichnet die verschiedenen Etappen seines Denkens recht deutlich nach und führt umgekehrt vor Augen, welch große Bedeutung wiederum der Reisebericht für einzelne Etappen seines Denkens hatte. Wie aber funktionalisiert und refunktionalisiert ein 'echter' Vertreter der Postmoderne, vielleicht sogar einer ihrer Vordenker, den Reisebericht gerade auch in Hinblick auf die amerikanische Welt? Das vielleicht beste Beispiel hierfür stammt von einem Philosophen, ebenfalls aus französischen Landen, Jean Baudrillard. Es ist das erstmals 1986 auf Französisch erschienene Bändchen Amérique, mit dem wir uns nun in der Vorlesung auseinandersetzen wollen.

Im Gegensatz zum Titel des Buches geht es nicht etwa, wie man vermuten könnte, um ganz Amerika, um den gesamten amerikanischen Kontinent, sondern lediglich um die USA, die diesen Begriff schon seit langer Zeit und mit zunehmendem Erfolg für sich beschlagnahmt haben. Das kennen wir schon: Es ist Teil der rhetorischen Selbstverständlichkeiten des modernen Diskurses über die Amerikas. Amerika wird gleichgesetzt mit den Vereinigten Staaten; und schon im ersten Kapitel erfährt man, warum Baudrillard sich im Zeichen der Postmoderne ausschließlich für die Welt der USA interessiert. Mit einem gewissen Recht könnte man ihm vorwerfen, bei seiner Globalisierungstheorie eine ausschließliche, vielleicht größtenteils auch unbewusste Reduktion auf die G7-Problematik zu betreiben. Doch davon gleich mehr, gehen wir zunächst einmal systematisch an diesen Text heran.

Gleich zu Beginn von Amérique sitzen wir natürlich in einem Auto, selbstverständlich von der Klimaanlage gekühlt. Damit haben wir bereits das bevorzugte Transportmittel von Baudrillards Reisebericht kennengelernt. Sein Buch beginnt mit einer Warnung: Die Dinge in diesem Spiegel könnten näher sein, als sie erscheinen! Dieses Motto ist recht ingeniös, spielt es doch gleich verschiedene Ebenen des Textes ein und bezieht diese aufeinander.

Zum einen wissen Sie ja, das auf allen verkleinernden Spiegeln in den USA diese Warnung aufgedruckt sein muss, um Fehleinschätzungen und daraus sich ergebende Schadensersatzklagen gegen die Hersteller aus dem Wege gehen zu können. Derartige Spiegel finden sich vor allem an Bord von privaten Automobilen, und damit wird von Beginn an das beliebteste Fortbewegungsmittel in den Vereinigten Staaten gleichsam von innen, vom Fahrersitz aus, in Szene gesetzt. 


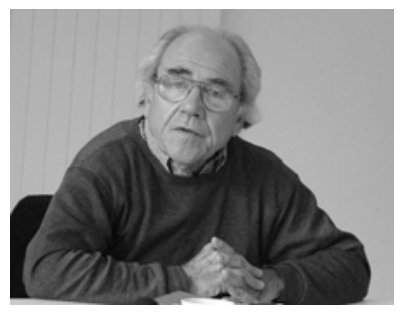

Abb. 92: Jean Baudrillard (Reims, 1929 - Paris, 2007).

Wir werden in diesem Buch nämlich auf ungezählten Highways und Freeways durch die Vereinigten Staaten geführt - und ich darf sagen, dass diese Vektorizität einerseits diskontinuierlich und andererseits ungerichtet ist. Wir haben es folglich mit einem weiteren Reisebericht der zweiten Hälfte des 20. Jahrhunderts $\mathrm{zu}$ tun, welcher durch die hermeneutische Bewegungsfigur eines diskontinuierlichen Springens gekennzeichnet ist. Weiß man denn, wohin die Reise geht?

Zum zweiten wird natürlich auch hier die Spiegelmetapher verwendet, die - wie Sie ja wissen - eine überaus lange Tradition in der abendländischen Kultur- und Literaturgeschichte besitzt. Die Metaphorologie des Spiegels gewann an (auch epistemischer) Schärfe insbesondere seit sie der französische Romancier Stendhal im Vorwort $\mathrm{zu}$ Le rouge et le noir verwendete und damit den mimetischen Abspiegelungscharakter seines Romans betonte. Denn er selbst sei nicht dafür verantwortlich, dass dieser Spiegel nicht nur den azurnen Himmel, sondern auch den Dreck am Straßenrand zeige. Dafür solle man gefälligst nicht den Autor des Romans, sondern jene verantwortlich machen, die für den Zustand der Straßen gerade stehen müssen. Und Straßen in der Form der Highways spielen in Baudrillards Amérique eine entscheidende Rolle.

Für Stendhal also war der Roman ein Spiegel, den man eine Straße entlang trägt: Die Bewegungsmetaphorik und insbesondere auch der Straßenverlauf sind als mobile Vektorizität damit in den Reisebericht eingeschrieben. Sie spielen selbstverständlich eine herausragende Rolle generell in der Reiseliteratur, einer Literaturgattung, die ja par excellence in Bewegung ist und sich an Land entlang von Straßen und Wegenetzen bewegt.

Mit dem Rückgriff auf die Spiegelung und deren metaphorisches Feld blendet Baudrillard zugleich aber auch seine Vorstellungen von unendlichen Spiegelungen ein, insoweit die Welt als Simulakrum, als eine Art von Spiegelkabinett verstanden werden kann, in dem die Ursprünge, die ursprüngliche Realität, verloren geht in einem ganzen Netzwerk an Substitutionen, die an die Stelle eigentlicher, authentischer, realer Erfahrung treten. Das Simulacrum ist eine zentrale Denkfigur und zugleich ein wichtiges Schreibverfahren von Jean Baudrillard, der sich auch in Amérique als Philosoph erweist. Wir genießen, um ein wenig zu 
übersetzen, was Baudrillard mit dem Simulakrum meint, nicht mehr die Erfahrung einer Welt der Realität, sondern die Erfahrung einer Welt der Zeichen, die für die Welt der Realität steht und diese simuliert, gleichsam an deren Stelle tritt. Dies wäre ein schöner Anfang für einen Roman, ist aber auch als Eingangstor in einen Text der Reiseliteratur und der Philosophie bestens geeignet. Denn wir treten ein - um es mit den Worten des Argentiniers Jorge Luis Borges zu sagen, der so etwas wie der Taufpate der Postmoderne ist - in einen Garten von Pfaden, die sich verzweigen.

Das erste Kapitel von Baudrillards Amérique ist mit Vanishing point überschrieben, was so viel wie im optischen Sinne Fluchtpunkt bedeutet, wörtlich übersetzt ein entschwindender Punkt, ein Punkt, der verschwindet. Bei Jean Baudrillard darf man wie überhaupt in der Postmoderne immer gespannt sein auf die Doppelt- und Dreifachbedeutungen, auf das, was Saussure die mots sous les mots nannte - womit er freilich eine anagrammatische Struktur meinte -, also auf die Polysemie, die Vieldeutigkeit der Worte, die jeweils in unterschiedlichen Sinnzusammenhänge andere, unabschließbare Sinnkomponenten und Sinnketten erzeugen. Wenn Sie so wollen, finden Sie auch auf dieser semantischen Ebene die Konzeption eines Kunstwerks als Mobile, das ständig in Bewegung ist und im Übrigen auch einen Betrachter braucht, der seinerseits mobil ist, so dass sich beide Pole unablässig in ihren wechselseitigen Bezügen räumlich verändern.

Der ganze Text - der übrigens in den Berliner Bibliotheken nicht in der französischen Originalausgabe, sondern nur in der Übersetzung vorhanden zu sein scheint - fängt an wie die Verkörperung des American Dream schlechthin. Hören wir uns also nach dem eingangs diskutierten Motto einmal das incipit dieses Textes an:

\begin{abstract}
Nostalgie née de l'immensité des collines texanes et des sierras du Nouveau-Mexique: plongées autoroutières et supertubes sur la stéréo-Chrysler et vague de chaleur - la photo ponctuelle n'y suffit plus - il faudrait avoir le film total, en temps réel, du parcours, y compris la chaleur insupportable et la musique, et se reprojeter tout cela intégralement chez soi, en chambre noire - retrouver la magie de l'autoroute et de la distance, et de l'alcool glacé dans le désert et de la vitesse, revivre tout cela au magnétoscope chez soi, en temps réel - non pour le seul plaisir du souvenir, mais parce que la fascination d'une répétition insensée est déjà là, dans l'abstraction du voyage. Le déroulement du désert est infiniment proche de l'éternité de la pellicule. ${ }^{1}$
\end{abstract}

Ein fürwahr dichter Anfang eines Textes, der es in sich hat und zugleich reiseliterarisch eine Abfolge von Herausforderungen darstellt, die wir uns in unserer

1 Baudrillard, Jean: Amérique. Paris: Grasset 1986, 9 f. 
Vorlesung zumindest exemplarisch näher ansehen müssen. Doch irgendwie wirkt alles auf uns vertraut: Die Chrysler-Stereoanlage, der kühle Alkohol in der Wüste, die Autobahnen und Highways, die Durchquerung der Wüste im Automobil. Irgendwie lässt dies alles in uns altbekannte Bilder hochsteigen, die immer wieder vor uns entrollt werden. Unsere Hypotypose entfaltet vor unserem inneren Auge die stereotypen Bilder einer Bilder-Sprache, zu welcher die Stereophonie den entsprechenden Sound liefert.

$\mathrm{Zu}$ diesen vorperspektivierten Erfahrungen tritt freilich eine Verdoppelung, nämlich die Verdoppelung der Erfahrung im Medium. Vielleicht ist hier auch das Medium die message. Dabei ist nicht nur die mittransportierte Musik, sondern - vergleichbar mit dem Blick aus dem Fenster eines fahrenden Autos - die Wirklichkeit des Films, genauer noch des Videos, neben die direkte Wahrnehmung von Realität getreten. Ist es die Welt, die an uns vorüberrauscht, oder ein Film? Dabei macht der letzte Satz klar, dass dieses Medium sich nicht etwa der Wirklichkeit annähert, sondern umgekehrt sich die Wirklichkeit - hier die Wüste - sich dem Film nähert, ihm dabei sogar recht nahe kommt. Was ist das Original, was ist die Kopie? Und gibt es einen Unterschied?

Damit ist bereits hier das Motiv der Spiegelung im Medium aufgenommen. Klar ist, dass hier nicht unbedingt ein Original existiert, das medial gespiegelt wird. Die Wüste ist vergleichbar mit einem langen Film, mit jenem Spiegel, auf dessen Fläche erst noch etwas gespiegelt werden wird. Sie entrollt sich vor uns, unendlich. Damit ist die Leere der Wüste hier gleichsam die Voraussetzung für jenes chemische Geschehen, das sich - damals noch zeitgemäß - in der Dunkelkammer abspielen wird - ein Phänomen der puren Medialität.

Im Grunde ließe sich bereits an dieser Stelle sagen, dass die eigentlichen postmodernen Landschaften jene zu sein scheinen, die sich im Westen und Südwesten der USA befinden. Sie bilden die eigentliche Projektionsfläche von Jean Baudrillards verschiedene Medien querender und daher unverkennbar transmedialer Reiseliteratur. Es sind die Wüstenlandschaften an der Grenze zu Mexico, die ähnlich wie in Michel Butors Experimentalroman Mobile gleich zu Beginn dieses Textes auftauchen und damit auch das Phänomen der Grenze aufrufen, ist hier doch mit Bedacht unmittelbar am Anfang der Name des Bundesstaates New Mexico eingeblendet. Darin liegt auch der Grund, warum ich im Anschluss an dieses erste Zitat im Fortgang das zweite Zitat, den zweiten Abschnitt des Buches, einblenden und Ihnen vorstellen möchte.

Les Mexicains devenus Chicanos servent de guides dans la visite d'El Alarno pour exalter les héros de la nation américaine si vaillamment massacrés par leurs propres ancêtres - ceux-ci 
ont fait le plus dur, mais ils n’ont pas échappé à la division du travail, aujourd'hui ce sont leurs petits-ftls et leurs arrière-petits-ftls qui sont là, sur le même lieu de bataille, pour exalter les Américains qui leur ont volé leur territoire. L'histoire est pleine de ruses. Mais les Mexicains aussi, qui ont passé clandestinement la frontière pour venir travailler ici. ${ }^{2}$

Damit ist das Phänomen der Grenze, wie schon in Michel Butors Mobile, von Beginn an in den Text von Amérique eingebracht. Es ist keine statische Grenze, die man etwa durch eine Grenzmauer befestigen könnte, sondern es sind mobile Borderlands, die eine eigene Literatur und Kunst hervorgebracht haben, von denen jedoch Baudrillards Text wenig weiß und denen wir uns daher auch nicht widmen wollen. Jedenfalls zeigt sich, dass diese Grenze gleich in mehrfacher Hinsicht beweglich ist. Denn zum einen ist sie dies in dem Sinne, dass hier einstmals - um die Mitte des 19. Jahrhundert - ein Kampf zwischen Amerikanern (in Baudrillards Diktion gleich US-Amerikanern) und Mexikanern stattgefunden hat, aufgrund dessen die Grenze weiter nach Süden verschoben wurde. Dies ist der Verweis auf die sogenannte guerra de rapiña, jenen Raubzug, den die US-amerikanische Armee gegen den Nachbarstaat Mexico in den 1840er Jahren durchführte.

El Alamo erinnert in diesem Sinne an Auseinandersetzungen, die schließlich zu jenem ungleichen Krieg führten, in dem Mexico mehr als ein Drittel, fast die Hälfte seines damaligen Staatsgebietes verlor und an die USA abtreten musste. Damit wird die Expansion der USA eingeblendet, das Vorschieben der Grenzen der Vereinigten Staaten nach Westen und nach Süden auf Kosten des mexikanischen Nachbars in einer Bewegung, die man sehr wohl als imperialistisch bezeichnen darf. Doch all dies ist zur Folklore, zu einem Fall für Fremdenführer geworden.

Zugleich gibt es aber wiederum eine andere Grenze, die nun im Bereich der Borderlands ständig überschritten wird, um gerade deshalb auch weiterhin als Grenze sichtbar zu sein. Diese Grenze zeigt sich auch im Bereich der kulturellen Alterität, die ebenfalls - erneut wie bei Michel Butors Mobile - von Beginn an präsent ist, eine kulturelle Alterität, die auch hier wieder aufgestellt und unterlaufen wird. Denn die mittlerweile zu Chicanos gewordenen Mexikaner bewegen sich nicht in der Spur ihrer Vorfahren - womit sie eine Art Fortführung von einem vermeintlichen 'Ursprung' her betrieben -, sondern nun im Dienste der ehemaligen Feinde. Deren Helden lassen sie nun, angestellt von den Vereinigten Staaten, hochleben, freilich nur, um gerade hiermit die Grenze umso besser unterlaufen zu können.

2 Ebda., S. 10. 
Wo ist hier in den Borderlands die Authentizität geblieben? Wo ist überhaupt das Schlachtfeld versteckt, der originäre Ort des Massakrierens, den der Reisende sucht und vermeintlich vorfindet? Mit Ausnahme seines Eigennamens, eines Wortes und damit eines Zeichens also, ist er verschwunden, zubetoniert und mit Blumen geschmückt. Hat es überhaupt je eine Schlacht gegeben in diesen Landstrichen, in denen sich Wüste und Film wie Film und Kopie gleichen? Authentizität ist also auch hier nicht zu finden, die Grenzlinien sind nicht klar gezogen, die Orte sind längst medialisiert und als solche hinter ihren Zeichen verschwunden. Die Zeichen sind an die Stelle der Authentizitäten, der Essentialitäten, getreten und nehmen die gesamte Breite des Bildes ein, das der Reisende vorfindet und beschreibt. Wir haben es mit einer Reiseliteratur in den Zeiten postmoderner Simulakra zu tun, einer Reiseliteratur, welcher der außersprachliche Gegenstand zunehmend entschwindet und zu einem vanishing point wird.

In der Folge läuft vor der Leserschaft gleichsam ein Film mehr oder minder bekannter Landschaften ab: Salt Lake City und seine Wüste, Monument Valley, wobei die Überreste dieses Naturschauspiels nicht mehr länger 'natürlich' wirken, geben sie doch laut Erzählerfigur die beste Vorstellung davon, was eine Kultur ist. Denn natürlich ist Natur nicht natürlich: Sie wird stets von der Kultur, von einem kulturellen Blickpunkt aus, entwickelt.

Stets präsent ist die Dimension der Zeit, die wir ja bereits im incipit von Baudrillards Amérique in der zweifachen Form der Perzeption und in jener der Geschwindigkeit kennengelernt haben. Dies hat gewiss auch mit dem genius loci zu tun, ist doch gerade die Wüstenlandschaft des nordamerikanischen Südwestens der privilegierte Ort für Hochgeschwindigkeitsversuche, jener Ort also, an dem die Menschen versuchen, auf der Erde die größtmögliche Geschwindigkeit zu erreichen und damit diese Geschwindigkeit in die Leere der Wüste einzuschreiben. Es geht nicht um das Amerika der Tiefe, es geht vielmehr um das Amerika der Oberfläche - und gerade dafür ist die Wüste in ihrer Flächenhaftigkeit bestens geeignet.

Denn alle anderen Landschaftsformen geben immer ein Gefühl der erdräumlichen, geologischen oder geomorphologischen Tiefe ab, nicht aber die Wüste, die gerade in ihrer Tiefe durch einen Mangel, ein Fehlen des Grundwassers, gekennzeichnet ist. Damit entbehrt sie das lebensspendende Element, das Wasser, das ihr einen organischen Charakter verliehen hätte, den sie aber ohne diese Dimension der Tiefe im Gegensatz zu anderen Landschaften nicht besitzt. Hier sehen wir die für die Postmoderne charakteristische Liebe zur Oberfläche und Abneigung gegenüber der Tiefe in einer ganz konkreten Landschaftsformation verkörpert: der flächenhaft sich erstreckenden Wüste, in die kein Pflug, keine Agrikultur eindringt. 
So verwundert es nicht, dass in Jean Baudrillards Reisebericht in der Folge das Hohelied der Wüste angestimmt wird und dass sich eine Folge von Lobeshymnen über die Wüste und die Reinheit ihrer Luft ergießt. Als eine Landschaft der Theorie ist die Wüste die eigentliche Landschaft der Postmoderne. Frühere Stadien, noch vor der Exterminierung der Wüstenindianer, werden nun sichtbar: Es sind geologische und mineralogische Stadien, also das, was in Baudrillards Reisebericht als das siderische Amerika bezeichnet wird. Hieran kann sich eine Theorie der Simulakren bevorzugt festmachen.

Neben der menschlichen Zeit und der Zeit der Geschwindigkeit werden damit weitere Zeitebenen, auf denen die Zeit langsamer abläuft, sichtbar: die Zeit der Gesteinsbildung und die Zeit der Auskristallisierung der Mineralien. Dabei existiere nirgendwo ein solches Schweigen, das deutlich posthumane Züge annimmt; und dieses Schweigen sei auch etwas Visuelles. In den Bergen, so heißt es, könne kein Schweigen sein, würden doch schon die Konturen der Berge brüllen. Die flache Oberfläche der Wüste aber bietet hierzu einen Gegensatz, der Stille ist.

Das Schweigen, so heißt es bei Jean Baudrillard noch immer im ersten Kapitel, muss eine Art von Horizontalität beinhalten. Das Sichtbarwerden der Mineralität und das Hinzutreten des Siderischen, damit folglich von Astroamerika, der Sterne also, die man ja bekanntlich auch aus Wüsten heraus am besten sehen kann, beruht freilich auf dem Verschwinden der Wüstenindianer, die - so heißt es im Buch lapidar - erst vernichtet werden mussten, um all dies sichtbar zu machen. ${ }^{3}$ Das Massakrieren der Wüstenindianer ist folglich die Voraussetzung für das Schweigen, die Stille.

Von großer Bedeutung - und dies soll das letzte Zitat aus dem ersten Kapitel Vanishing Point sein - ist dabei die Geschwindigkeit, die gleichsam die Objekte selbst transformiert und alle Formen von Wahrnehmung verändert:

La vitesse est créatrice d'objets purs, elle est elle-même un objet pur, puisqu'elle efface le sol et les références territoriales, puisqu'elle remonte le cours du temps pour l'annuler, puisqu'elle va plus vite que sa propre cause et en remonte le cours pour l'anéantir. La vitesse est le triomphe de l'effet sur la cause, le triomphe de l'instantané sur le temps comme profondeur, le triomphe de la surface et de l'objectalité pure sur la profondeur du désir. La vitesse crée un espace initiatique qui peut impliquer la mort et dont la seule règle est d'effacer les traces. Triomphe de l'oubli sur la mémoire, ivresse inculte, amnésique. Superfiàalité et réversibilité d'un objet pur dans la géométrie pure du désert. Rouler crée une sorte d'invisibilité, de transparence, de transversalité des choses par le vide. C'est une sorte de suicide au ralenti, par l'exténuation des formes, forme délectable de leur disparition. La vitesse n'est pas végétative, elle est plus proche du minéral, d'une

3 Ebd., S. 15.18 
déflection cristalline, et elle est déjà le lieu d'une catastrophe et d'une consumation du temps. Mais peut-être sa fascination n'est-elle que celle du vide, alors qu'il n’y a de séduction que du secret. ${ }^{4}$

Was für ein Text und was für ein Reisebericht, der dieses Lob der Geschwindigkeit im Sinne einer Auslöschung der Spuren, einer Auslöschung der Geschichte, einer Auslöschung der Zeit als vanishing point zelebriert! Das ist in der Tat recht starker Tobak, der zugleich in der Hervorhebung aller Oberflächen jene Tiefe abschafft, welche der Seinsgrund der europäischen Moderne war: jener Tiefeder marxistischen Analyse des ökonomischen Unterbaus, jener Tiefe im Freud'schen Modell des Psychischen Apparats. In dieser amerikanischen Landschaft der Theorie wird ein Epochenwechsel proklamiert mit aller programmatischen Entschlossenheit, derer der französische Philosoph fähig ist.

Denn im Grunde werden in diesem Lob der Geschwindigkeit und der Wüste jene Grundregeln und Grundgesetze außer Kraft gesetzt, die letztlich die modernen Naturwissenschaften wie auch die modernen Geisteswissenschaften beherrscht haben und - so darf beschwichtigend hinzugesetzt werden - wohl auch noch immer beherrschen. Denn der normalisierte Ablauf von der Ursache zur Wirkung, also das Kausalitätsprinzip wird hier ebenso unterminiert wie das Denken in der Tiefe, das ja die Diskurse einer Vielzahl moderner Theorien oder - wie Lyotard sagen würde - grands récits geprägt hat: Denken Sie nur an die Psychoanalyse, die unter der Oberfläche des Manifesten stets das Latente, unter der Oberfläche einer Handlung oder eines Traumes stets die frühkindlich-geschichtliche Herausbildung suchte, um von der Wirkung an der Oberfläche auf eine Ursache in der Tiefe verweisen zu können. Denken Sie an den marxistischen Materialismus, der alle Oberflächenphänomene in einer überaus komplexen Theorie stets und unentrinnbar mit der Basis, dem Unterbau, verband und gleichsam metaphorisch hübsch als Überbauphänomene letztlich auf das Fundament der Architektur zurückschraubte. Denken Sie aber auch an das Christentum, das die verschiedensten Formen der Oberfläche stets auf die Grundstrukturen von Gut und Böse zurückführte, konnte doch auch und gerade der Teufel in den Formen der Verführung erscheinen. Dies sind nicht mehr und nicht weniger als die Grundlagen des abendländischen Denkens. Wie bei Roland Barthes also eine dépossession de l'Occident?

Sie merken, wie viele Dimensionen der oben zitierte Text hier enthüllt: Eine ganze Philosophie steht dahinter, welche versucht, die Grundfundamente der abendländischen Philosophie zu unterminieren, in die Luft zu jagen - oder ganz einfach an ihnen im Reisebericht vorbeizufahren. Aber lässt sich dies so einfach bewerkstelligen?

4 Ebda., S. $16 \mathrm{f} ., 19 \mathrm{f}$. 
Die Geschwindigkeit schafft ihre eigene Zeitlichkeit - was jedem, der einmal ein paar Stunden lang schnell in einem Auto gefahren ist, bekannt ist, und sie schafft auch ihre eigenen Denkhorizonte. Sie merken auch: Das Lob der Oberfläche ist in keiner Weise gleichbedeutend mit einer Oberflächlichkeit des Denkens. Was in diesen Reflexionen entwickelt wird, ist eine Art Gegenwelt zu einer in Kausalitätsketten erstarrten, immer wieder die historische Tiefe zur Erklärung der aktuellen Oberfläche aufsuchenden Denkens, gegen das Baudrillard hier ganz einfach die Erfahrung - man könnte sagen: die lustvolle Erfahrung auch im automobilistischen Sinne - der Oberflächen setzt.

Eine große Verführungskraft scheint freilich davon auszugehen, auch wenn im Zentrum dieses Denkens und dieser Vorstellungen kein eigentlicher Kern, jener Nucleus, der den grands récits ihre Tiefe und Dichte gab, sondern vielmehr ein leeres Zentrum sichtbar wird. Es erinnert uns ganz deutlich an jenes centre ville centre vide, das in Roland Barthes' L'Empire des signes bei der Darstellung von Tokio entfaltet wird. Gegen das gefüllte wird ein entleertes Zentrum gesetzt, so wie auch bereits der Pariser Eiffelturm für Barthes ein leeres Zentrum in seiner filigranen Eisenstruktur umschließt. Dies sind die neuen (urbanen) Landschaften, welche der Reisebericht der Postmoderne programmatisch in Stellung bringt. Die historische Akzeleration der Moderne, die Beschleunigung der geschichtlichen Zeit, die als Zeiterfahrung seit der Französischen Revolution den Zeitgenossen und bis in unsere Zeit hinein bekannt ist, hat nun so viel Fahrt aufgenommen, dass diese Geschwindigkeit ihre eigene Zeitlichkeit und ihre eigene Objektwelt herstellt. Denn der Zeitpfeil ist fortan allen Gegenständen eingeschrieben und verändert deren Perzeption.

Wir finden in diesem literarischen Reisebericht von Jean Baudrillard eine ganze Reihe von Theoremen postmodernen Denkens. So sei die Gesellschaft der Zukunft eine Gesellschaft der Komplexität, der Hybridität mit einer Geschlechtlichkeit von unvorhersehbaren Konsequenzen. Die Wunder finden in den künstlichen Paradiesen statt, die sich die Menschheit geschaffen hat. Gegen das Reisen im Sinne von Tourismus und Ferienreisen setzt Baudrillard ein reines Reisen, das sich keine Ziele vorgibt, sondern einfach in der Bewegung die eigene Motivation im doppelten Wortsinne hat. Daher könne man auch am besten im Raum der Wüste reisen: oder auch in einer nicht weniger wüstenhaften Anlage, im Raum einer großen Metropole oder mit anderen Worten: in einer urbanen Landschaft der Theorie.

Dies ist zweifellos ein wichtiger Aspekt der Behandlung des urbanen Raumes, der Annäherung an die Großstadt, die in diesem Text selbstverständlich keineswegs ausgeblendet, sondern als anderer Bezugspunkt auftaucht. Doch Wüste und Stadt, menschenleerer und menschenüberfüllter Raum stimmen miteinander überein in einer bestimmten Konzeption von Raum und Zeit. Die 
Deterritorialisierung, so Baudrillards Erzählerfigur, befreie uns. Das Reisen wird hier auf Bewegung reduziert, das Autofahren erscheint explizit als eine spezifische Form des Gedächtnisverlustes. Diskontinuierliche Sprünge, im Hier und Jetzt der Oberflächen ausgeführt in unterschiedlichste Richtungen: Das ist die Amérique zugrunde liegende hermeneutische Bewegungsfigur. Es soll Leute geben, die mit ihrem Auto über Autobahnen jagen, um diese Art von Gedächtnisverlust künstlich zu erzeugen, um diesem eigenen Gedächtnis ein Vergessen entgegensetzen zu können, ein Vergessen jener Last, welche Tiefe mit sich bringt. Eine Moral scheint es in Baudrillards Text aber doch auch zu geben: 'Morgen ist der erste Tag des Restes Deines Lebens'. Sie merken: keine Reise ohne ihre eigene (Reise-) Philosophie.

So kann es uns nicht überraschen, dass uns nach dem ersten Kapitel, in dessen Mittelpunkt die Wüste steht, nun im zweiten Kapitel eine Stadt entgegentritt, was sage ich, die Metropole schlechthin, New York. Im Sinne Baudrillards die Stadt der Städte: New York ist die Stadt, die für alle anderen Städte von Bedeutung ist. Ein Besuch in der Stadt ist eine Annäherung an das Zentrum der Welt. Wie bitte? Also hat die Welt doch noch ein Zentrum?

Schauen wir uns das Leben in New York - bisweilen aus dem Hotelzimmer gesehen - einmal mit Hilfe dieses Reiseführers aus der Feder Baudrillards genauer an.

Pourquoi les gens vivent-ils à New York? Ils n'y ont aucun rapport entre eux. Mais une électricité interne qui vient de leur pure promiscuité. Une sensation magique de contiguïté, et d'attraction pour une centralité artificielle. C'est ce qui en fait un univers auto-attractif, dont il n'y a aucune raison de sortir. Il n'y a aucune raison humaine d'être là, mais la seule extase de la promiscuité. [. . .] La merveille des démolitions modernes. C'est un spectacle inverse de celui d'un lancement de fusée. Le building de vingt étages glisse tout entier à la verticale vers le centre de la terre. [...] Voilà un art merveilleux de la modernité, qui égale celui des feux d'artifice de notre enfance. [...] A New York, le tournoiement de la ville est tellement fort, la puissance centrifuge est telle qu'il est surhumain de penser vivre à deux, de partager la vie de quelqu'un. Seuls, les tribus, les gangs, les mafias, les sociétés initiatiques ou perverses, certaines complicités peuvent survivre, mais pas les couples. C'est l'anti-Arche, où les animaux étaient embarqués par deux pour sauver l'espèce du déluge. Ici, dans cette Arche fabuleuse, chacun est embarqué seul - c'est à lui de trouver, chaque soir, les derniers rescapés pour la dernière party. [...] Je n'aurais jamais cru que le marathon de New York puisse vous arracher des larmes. C'est un spectacle de fm du monde. [.. .] Sous la pluie battante, sous les hélicoptères, sous les applaudissements, revêtus d'une capuche d'aluminium et louchant sur leur chronomètre, ou torse nu et les yeux révulsés, tous cherchent la mort, la mort par épuisement qui fut celle du marathonien d'il y a deux mille ans, qui, ne l'oublions pas, portait à Athènes le message d'une victoire. ${ }^{5}$

5 Ebda., $35 \mathrm{ff}$. 
Diese Szenen aus New York, zusammen mit vielen anderen, die ich Ihnen hier nicht darstellen kann, zeigen eine Stadt, die irgendwie an einem Ende oder an einer Grenze angekommen zu sein scheint: an einem Punkt der Erschöpfung. Die Apokalypse ist da, und irgendwie hat es keiner gemerkt. Jean Baudrillard besingt in diesem Reisebericht die Apokalypse, beschwört ihr Kommen. Es gilt an dieser Stelle darauf aufmerksam zu machen, dass das Thema der Apokalypse in der Tat ein für die Postmoderne wichtiges Bezugsthema ist, dass sie aber nicht in der moralischen Härte auf die schuldig gewordene Menschheit herniedergeht, sondern in einer Art sanftem Regen der Lust, wo die Grenzen der Katastrophe nicht sichtbar sind - selbst nachdem sie sich ereignet hat. Schon die Darstellung des Zusammenbruchs ist lustvoll. Und Baudrillard frönt dieser Lust am Schauspiel des Zusammenbruchs in vollen Zügen.

Dabei ist das Zeitgefühl und die Katastrophenstimmung nicht unbedingt die eines Bruches, wohl aber die Betrachtung aus der Perspektive einer Nachzeit, die es erlaubt, das Vergangene neu zu perspektivieren und an seinem Untergang, der auch der eigene Untergang nebst Zukunftsversprechen ist, als Zuschauer teilzunehmen. Als Zuschauer eines Schiffbruchs, wie Hans Blumenberg gesagt hätte. ${ }^{6}$ Diese Zuschauerperspektive ist es, die letztlich alle Geschehnisse, auch die eigene Reise, wie in einem Film, in einem anderen Medium erleben lässt. Amérique von Jean Baudrillard ist in dieser Hinsicht vielleicht die postmodernste aller Reiseunternehmungen am Ausgang dieses Jahrhunderts. Genau dies macht auch den hohen Reiz dieses Reiseberichts aus, vermittelt er uns doch ein Gefühl für jene Stimmung, die sich im letzten Drittel des 20. Jahrhunderts sowie gegen Ende dieses Jahrhunderts der Weltkriege gesamtgesellschaftlich zumindest in Europa und in den Vereinigten Staaten breit machte.

Denn eine Fin de siècle-Stimmung lässt sich in diesem Text sehr wohl ausmachen. Es ist die Lust, am Ende eines Jahrhunderts, am Ende einer Zeit, am Ende eines Jahrtausends zu leben und dem langsam vergehenden Zeitraum den Rücken zuzuwenden. Mit Blick worauf? Die Gesten, die uns die Antike mit auf den abendländischen Weg gab, sind noch vorhanden, wie der Marathonlauf zeigt. Aber eben nur noch die Gesten, nur noch die Oberflächen, nur noch das Spektakel, das in sich selbst, in der Tiefe, des Grundes verlustig gegangen ist. Dem Verlust der Tiefe, der tiefgreifenden Motivation, entspricht die Schönheit der Oberflächen, die szenisch herausgeputzt brillieren.

Ist die Welt an einem Ende? Und wird dieses Ende mit einer Lust zelebriert, die aus der heutigen Perspektive doch erstaunen mag? Nein, es ist keine Tötung,

6 Vgl. Blumenberg, Hans: Blumenberg, Hans: Schiffbruch mit Zuschauer. Paradigma einer Daseinsmetapher. Frankfurt am Main: Suhrkamp 1979. 
sondern die Selbsttötung, es ist kein Untergang, sondern schlicht die Erschöpfung - eine Deutung, die an John Barth und seine Poetik der exhaustion, der Entkräftung und Erschöpfung, erinnert. Versuchen wir aber abschließend, uns noch mit einigen für unsere Vorlesung wichtigen Aspekten zu beschäftigen, Aspekte, die insbesondere auch die Frage der kulturellen Alterität betreffen. Denn auch hier eröffnet Jean Baudrillards Reisebericht eine Vielzahl aufschlussreicher Perspektiven und Ausblicke.

Der reiseliterarische Text des Franzosen Baudrillard funktioniert in gewisser Weise trotz all seiner Ausfälle gegen binäre Logiken nach dem Prinzip eines Zweitaktmotors, der ständig die Situation in den USA durch den europäischen Blickwinkel kontrastiv ergänzt. Das den gesamten Text prägende Spannungsfeld ist das zwischen Europa und den Vereinigten Staaten von Amerika. Einen neuen Blickwinkel auf Europa könne man, entgegen aller Erwartungen, freilich von den USA aus nicht gewinnen; eine neue Perspektive auf Europa könne man nur in Europa entwickeln. Gehe man von Europa in die USA, so verschwinde Europa einfach. Auch hier ist es die Lust an einem Verschwinden, an einem fast lautlosen Aufhören von etwas, das man die eigene Herkunft des französischen Philosophen nennen darf. Aber sehen wir uns dieses Verschwinden etwas genauer an.

Amerika sei ein gigantisches Hologramm in dem Sinne, dass es jede Information über das Ganze in jedem seiner Teile enthält, so der Erzähler des Reiseberichts. Nehme man ein einzelnes Element wie etwa MacDonald, eine Studebaker oder sonst etwas heraus, so enthalten diese einzelnen Elemente stets das ganze Amerika in sich. ${ }^{7}$ Gleichwohl bleibt das Problem der Beziehung zu Europa in Baudrillards Text allgegenwärtig. Denn Baudrillard rekurriert dabei immer wieder auf Schlagwörter, für die er in seinen theoretischen Schriften zum Teil auch selbst verantwortlich zeichnet, so wie etwa Hyperrealismus, alles sei Simulacrum und Simulation sowie vieles mehr. So geistert auch das Thema der Apokalypse immer wieder durch diese Zeilen; so evoziere nichts deutlicher das Ende der Welt als ein Mensch, der geradeaus an einem Strand joggt; Primitive würden Selbstmord begehen, indem sie weit hinaus schwimmen ins offene Meer, der Jogger aber begeht durch sein Laufen strandauf strandab Selbstmord auf Raten. ${ }^{8}$

Halte man diesen Jogger aber an, so tanze er weiter vor einem hin und her wie ein Besessener. Das ständige Speichern von Informationen über die eigene Geschichte ist ebenso ein Thema wie die Problematik einer hypergeschützten Gesellschaft, in der wir kein Verhältnis mehr zum Tod, keine Achtsamkeit mehr

7 Vgl. Baudrillard, Jean: Amérique, S. 59.

8 Vgl. ebd., S. 75 f. 
besitzen trotz der Allpräsenz der Apokalypse in der Form der Star Wars und anderer Kriegsspiele. Überall ist die Orgie vorbei, wir leben in einer postorgiastischen Gesellschaft, so der Befund des Philosophen. Was aber kann die Zukunft einer Gesellschaft sein, so fragt man sich etwas ratlos, in der es keine Orgien mehr gibt?

Auch im Geschlechtlichen wird ständig die Frage der Grenzen gestellt, seien doch Typen entstanden, die gleichsam die beiden Geschlechter in der Massenkommunikation wieder zusammenführen. Nein, von einer Diversität der Geschlechter spricht Baudrillards Figur des Reisenden nicht, wohl aber ist ihm die Sicherheit einer binären Zuordenbarkeit abhanden gekommen. Doch der philosophe und voyageur demontiert nicht zuletzt das Bild, dass Amerika nett zu uns, nett zu Tieren und nett zur Welt sei, und evoziert die Bilder der Kriege und Kriege. Es brauchte kein WikiLeaks, um den französischen Philosophen über diese Seite der USA und ihrer hemmungslosen Kriegsführung in Kenntnis zu setzen.

Längst habe in 'Amerika' das Fernsehen den Platz des Chores in der griechischen Tragödie eingenommen. In anderen Ländern überlasse das Fernsehen das Lachen den Menschen, hier aber ist das Lachen in das Fernsehen und in die Show integriert. Und so ist es der Fernsehbildschirm selbst, der eine 'good time' hat, während wir alleingelassen werden mit den Bildern der Fernsehkriege. Nicht umsonst verweist Baudrillard auf das Apocalypse Now von Coppolas Vietnam-Film. er hätte auch auf Neil Postmans in den ausgehenden achtziger und beginnenden neunziger Jahren weit verbreitete Analyse Amusing Ourselves to Death hinweisen können. ${ }^{9}$

Wie Michel Butor in seinem Experimentalroman Mobile fliegt auch Baudrillard mit einem Text über Los Angeles, freilich des Nachts und auf ein Lichtermeer herunterblickend. Er macht aus dieser Perspektive schon im Flackern der Lichter den grundlegenden Unterschied zu den europäischen Städten deutlich. Und es kommt ganz so, wie es nicht anders zu erwarten war: Los Angeles sei ein bewohnter Teil der Wüste, die Freeways verlaufen einfach durch die Landschaft und die Stadt. Die Städte wurden vor den Freeways gebaut, und doch sieht es heute so aus, als ob sie entlang der Freeways gebaut worden wären.

Dasselbe geschehe auch mit der US-amerikanischen Realität insgesamt: Sie bestand sehr wohl, bevor die Bildschirme erfunden wurden; doch sieht es heute so aus, als sei sie erfunden worden im Gedanken an die Bildschirme. ${ }^{10}$ Daher sehe die US-amerikanische Stadt heute so aus, als ob sie gerade aus dem Film

9 Vgl. Postman, Neil: Amusing Ourselves to Death. Public Discourse in the Age of Show Business. London: Heinemann 1986.

10 Vgl. Baudrillard, Jean: Amérique, S. 109. 
käme, so als ob man in Cinecittà noch die letzten Kulissen aufgeboten habe. Alles ist mit allem verbunden, so wie Baudrillard die postmoderne Architektur sieht, bei der er fragt, ob es sich überhaupt noch um eine Architektur handele. In gewisser Weise versucht auch Baudrillards Buch, dem Motto, alles mit allem zu verbinden, gerecht $\mathrm{zu}$ werden. So bleibt auch die mystische Erfahrung nicht draußen: Sie erscheint gleichsam epiphanieartig in der Erfahrung von Death Valley, dem nichts Funebres, wohl aber etwas Wildes anhafte. Hier werden das Schweigen und die Stille wieder hörbar, das Schweigen der Zeit. Und auch hier erhält die Wüste einen kinematographischen Grundzug, der alles wie in einem Film vorüberrollen lässt. So erscheint die Ermordung der Indianer auch nur noch durch die Referenz gegenüber John Ford, von dem wir aus anderer Quelle ja wissen, dass er einmal von sich sagte, niemand habe mehr Indianer umgebracht als er selbst.

Im vierten Kapitel geht es um Amerika als verwirklichte Utopie: Hier erscheinen die USA als die ursprüngliche Version von Moderne und mehr noch Modernität. Europa aber wirkt aus dieser Sicht nur als die synchronisierte oder untertitelte Fassung dieser eigentlich nordamerikanischen Modernität. Amerika besitze, so der Reisende weiter, keine eigentliche Identität, besitze eigentlich keinen Ursprung, sondern lebe in einer beständigen Simulation, das heißt in einer ständigen Präsenz der Zeichen. ${ }^{11}$ Sind das wahre Reich der Zeichen folglich die USA? Wohl kaum. Dabei greift Baudrillard im Grunde auf das althergebrachte Modell zurück, demzufolge die USA das darstellen, was sich in Europa später erst entwickeln werde.

Dies ist ein Modell, das wohl erstmals in der französischen Literatur und Rechtsphilosophie entwickelt wurde und - wie wir bereits sahen - mit Alexis de Tocqueville wohl den ersten herausragenden Vertreter fand: Dort erschien die Démocratie en Amérique in gewisser Weise zukunftsschwanger für die künftige Entwicklung Europas. In diesem Kraftfeld bewegt sich auch Baudrillards reiseliterarischer Text. Die Macht der Vereinigten Staaten von Amerika beruht nicht zuletzt darauf, dass die Amerikaner daran glauben, die verwirklichte Utopie zu sein, und sie zugleich wissen, dass auch die anderen daran glauben.

So gebe es eine Faszination durch die USA auf der ganzen Welt, selbst bei jenen Völkern und Nationen, die unter den USA leiden. Allerdings werden wir, das heißt die Europäer, niemals die Naivität des Glaubens der Nordamerikaner an diesen Traum besitzen, so dass wir sie letztlich nur imitieren könnten. Amerika sei geschaffen worden, um aus der Geschichte zu entweichen; dieses Projekt werde heute noch immer weiterverfolgt. Wir hätten lange genug in dieser Modernität gelebt, um den Bruch durch die radikale Modernität der USA ermessen und

11 Vgl. ebda., S. $151 \mathrm{f}$. 
begreifen zu können. Nur in den Vereinigten Staaten sei man in der Lage, diese Modernität original $\mathrm{zu}$ leben und zugleich auch $\mathrm{zu}$ dezentrieren. ${ }^{12}$ Wir sehen: Immer wieder entwickelt Jean Baudrillards Erzählerfigur seine Gedanken anhand des Zweitaktmodells Europa versus Vereinigte Staaten und situiert alle Unterschiede als Gegensätze innerhalb dieses Spannungsfelds. Mit dem Aufstieg Amerikas begann Europa zu verschwinden; und alle Mythen seien heute amerikanisch, doch würde es uns nicht guttun, uns darüber zu ereifern und ärgern. In Los Angeles jedenfalls sei Europa verschwunden.

Kalifornien erscheint Baudrillard als die dezentrierte Antithese eines zentrierten Europa, wobei für den französischen Reisenden Europa mehr oder minder mit Frankreich in eins fällt oder gleichbedeutend ist. Der Mythos Amerika ist überall in Amérique spürbar. Auf einem Freeway in Kalifornien fragt sich der Erzähler, wie man eigentlich Europäer sein könne, ganz so, wie sich bei Voltaire die nicht ganz unhintergründige Frage erhob, wie man eigentlich Chinese sein könne. Jean Baudrillard malträtiert Europa ganz bewusst und explizit. Aber dabei belässt er es nicht. Denn das fünfte Kapitel von Amérique ist dem Ende der amerikanischen Macht gewidmet, wenn dies auch mit einem Fragezeichen versehen wurde.

In den Vereinigten Staaten seien die fünfziger Jahre die Zeit der großen Machtentfaltung gewesen; in den siebziger Jahren sei die Macht noch immer da und gegenwärtig, doch irgendetwas sei gebrochen. Die Orgie sei folglich vorbei. Die USA hätten heute an Macht eingebüßt und zwar aus dem schlichten Grunde, weil es heute kein Zentrum mehr gebe. Man könnte dies als naiv oder als wishful thinking bezeichnen. Mit Ronald Reagan und seiner Präsidentschaft sei ganz Amerika kalifornisch geworden im Sinne eines easy living. Kalifornisch sei es, die USA als die erreichte Utopie des Guten, die Sowjetunion - die es damals ja noch gab - als die erreichte Utopie des Bösen darzustellen. So einfach.

Politische und kommerzielle Werbung stimmen letztlich, so Jean Baudrillards Analyse, miteinander überein. Die Dummheit spiele keine Rolle, allein das Bild zähle: Man müsse nur glaubwürdig bleiben in einem System der Simulation von Regierung. Die Dritte Welt werde einfach verdeckt, lange lebe die Erste Welt, so wird der Reagan-Diskurs subsummiert. Und alles andere werde einfach in die Vergangenheit verdammt.

Wie dem auch immer sei: Für Baudrillard (oder die Stimme seines Erzählers) ist es unentscheidbar, ob die USA heute noch Macht besitzen oder Macht simulieren. Das ist freilich keine Frage, die für ihn ein grundsätzliches philosophisches Problem aufwirft. Die maschinenhaften Bewegungen, die an der Oberfläche sichtbar sind, könnten vielleicht doch besser funktionieren als die

12 Vgl. ebda., S. 161. 
lebendigen, da mit dem Leben auch die Probleme verschwunden seien. Amerika verfüge heute über eine Macht, die auf dem Werbebild beruht.

An all dies schließt sich das Bild eines möglichen Zusammenbruchs der Weltmacht USA an. Sie können aus dem zuvor gesagten bereits ableiten, wie diese Katastrophe aussehen wird; ich möchte Ihnen aber dennoch einmal abschließend vorführen, wie sich Baudrillard und sein Erzähler das Ganze ausgedacht haben:

C'est là la véritable crise de la puissance américaine, celle d'une stabilisation potentielle par inertie, d'une assomption de puissance dans le vide. Elle s'apparente sous bien des aspects à la perte des défenses immunitaires dans un organisme surprotégé. C'est pourquoi Reagan atteint du cancer me semble d'une ironie poétique. La figure du cancer est un peu à l'image de cette crédibilité transparente, de cette euphorie d'un corps qui ne produit plus d'anticorps, menacé de destruction par excès de fonctionnalité. Le chef de la plus grande puissance mondiale atteint du cancer! Le pouvoir saisi par les métastases! Les deux pôles de notre civilisation se rejoignent. Levée de l'immunité présidentielle, bientôt le SIDA! Ça devrait marquer le début de l'implosion générale (à l'Est il Y a longremps que le pouvoir est saisi par la nécrose). ${ }^{13}$

Auch wenn diese Analyse am Ende des Kapitels wieder etwas relativiert wird: Hier wird deutlich, wie Macht endet: in der Erschöpfung der Macht. Krankheiten gab es immer schon, die Abwehrkräfte aber sind nun geschwächt. Dass dies gerade nach einer Krankheit schreit, die eben die Abwehrkräfte sozusagen als Immunschwäche institutionalisiert, ist noch das letzte Tüpfelchen auf einer Interpretation, die sich radikal postmodern gibt. Und doch fällt sie letztlich immer wieder in einen Zweierrhythmus zurück, womit sie unentrinnbar bipolar organisiert und damit letztlich noch immer modern ist.

Die Metaphorik der Krankheit könnten wir an dieser Stelle verlängern und etwa mit Susan Sontags Aids as Metaphore weiterspinnen. Klar ist aber, dass die Postmoderne aus dem Gefühl der Nachzeit auch die Zeit des danach nicht nur instrumentalisiert, sondern vor allem inszeniert: als eine mehr oder minder lautlose Implosion, als ein Zerfallen, das nicht weit entfernt ist vom Zerfallen des Römischen Reiches, vom Gefühl, am Ende der Dekadenz und des Niedergangs der Reiche zu stehen. Die Postmoderne geht hier mit der Stimmung des fin de siècle einen lustvollen Pakt ein, der aber seit geraumer Zeit endgültig der Vergangenheit zuzugehören scheint. Denn auch Amérique von Jean Baudrillard ist längst wiederum historisch geworden. Und doch wirft nicht nur ihr Historisch-geworden sein, sondern gerade ihr historisches Gewordensein Probleme

13 Zitat 126: Jean Baudrillard: Amérique, S. $232 \mathrm{f}$. 
auf, die aufzunehmen und auszudenken auch für unsere Zeit höchst herausfordernd ist.

Im letzten Kapitel von Amérique wird das Lied der Wüste forever besungen, wobei der Amerikareisende nun die Problematik der Indifferenz innerhalb der neuen gesellschaftlichen Kontexte stark hervorhebt. Erneut werden die Großstadt und die Wüste in ein gemeinsames Bild transportiert, in das Bild einer Oberflächenkultur, die von der Tiefe befreit ist und damit auch von der Last jeglicher Geschichte. Am Ende dieses Buches steht das Verschwinden der Ästhetik, ein Verschwinden der Vieldeutigkeit der Kultur und ihrer Mehrschichtigkeit. Das einzige Gewebe der Städte seien die Freeways, mit ihren Abertausenden von Autos, die mit ihren Lichtern von nirgendwoher kommen und nirgendwohin gehen. auch hier ist die Vektorizität wiederum eine ungerichtete, eine zugleich multidirektionale, die nach unterschiedlichsten Richtungen gleichzeitig strebt.

Die Städte besitzen kein Zentrum mehr: Sie sind eine diskontinuierliche Abfolge von Zeichen ohne Hierarchie, ohne zergliedernde Ordnung und Rang. Am Ende des Buches finden wir das Lob von Kalifornien, seiner Diskonnektion zwischen städtischen Räumen und Wüsten, seiner Deterritorialisierung: Die Grenze der Wüste sei in diesem Zusammenhang initiatorisch. Im Grunde behandelt hier Baudrillard die Frage der Grenze zwischen Ökumene und Anökumene, wobei letztere durch eine Infrastruktur erschlossen wird, die zugleich die Anökumene transitabel macht und an die Zirkulation in den Städten anschließt. Dabei handelt es sich um eine Zirkulation und Kommunikation an der Oberfläche, auf der Oberfläche: Nichts davon erschließt die Tiefe. Die Last dieser Tiefe, dieser Geschichte, dieser Erinnerung, dieses Urgrundes wird durch eine List bewerkstelligt, einfach die Oberflächen zu bearbeiten, wodurch jene Lust entsteht, welche das Ich in seinen Bewegungen quer über die Oberfläche bereits seit den ersten Zeilen von Amérique durcherlebt.

Ganz am Ende des Bandes findet sich dann die Assoziierung des Spiels und von Las Vegas mit der Wüste, seien beide Räume doch erinnerungslos. Die Wüste besitzt die außergewöhnliche Bedingung, dass die Seltenheit von Zeichen sehr hoch wird, eine Konstellation, welche die Menschen dazu bringe, im Spiel die Augenblicklichkeit zu suchen. Mit diesen Schlussworten wird erneut die Oberfläche, die Unmittelbarkeit, die Vernichtung der historischen Tiefe, die Flucht aus der Abfolge langer Kausalketten evoziert, und all das abgeschüttelt, was unsere Entscheidungen vielschichtig, komplex, historisch abhängig und determiniert macht. Letzterem setzt Baudrillard die Wüste als Raum unsteter Bewegung, als Freiheit des Freeways, als Ziellosigkeit der Bewegungen und Bewegungsrichtungen entgegen. Dies bedingt dann letztlich auch die Grundstruktur seines Reisebuches, das ganz gewiss kein Reiseführer, sondern im besten Sinne Literatur, Reiseliteratur, ist. 
Die Realität, so predigte Jean Baudrillard in einem seiner berühmtesten Texte über das Simulakrum, gehe im Hyperrealismus, also in der exakten Verdoppelung des Realen, unter. Es verflüchtige sich von Medium zu Medium, werde zugleich aber zum Realen schlechthin. Der Widerspruch zwischen Realem und Imaginärem, so Baudrillard, sei im Hyperrealismus ausgelöscht. So sei die wirkliche Definition des Realen die, dass man von etwas eine äquivalente Reproduktion herstellen kann. Am Ende dann sei das Reale nicht mehr das, was reproduziert werden kann, sondern das, was immer schon reproduziert ist oder reproduziert worden ist.

Ursprung und Ziel, so Jean Baudrillard weiter, werden in ihrer Gegensätzlichkeit aufgehoben und durch die Verdoppelung ersetzt. Das Reale wird damit tendenziell vom Imaginären beziehungsweise vom Zeichenhaften ununterscheidbar. Der menschliche Raum wird damit gleichsam entrealisiert. Im Gegensatz zu den früheren Simulakren wie etwa jenen der Kunst oder des Spiegels sind die aktuellen Simulakren nicht mehr einfach und durchschaubar. Die Verschmelzung von Realem und Imaginärem bedeute letztendlich, dass überall die ästhetische Faszination herrsche. Alles, auch weit außerhalb der Kunst, werde im Zeichen einer derartigen Entwicklung ästhetisch. Allem liegt der Simulationsprozess zugrunde. Vor diesem Hintergrund ließe sich fragen: Ist nun Amérique eine derartige Simulation Amerikas? Die einfache Antwort auf diese Frage: wohl eher nicht. In jedem Falle ist Amérique eine Simulation geringeren Grades als Michel Butors experimenteller Roman Mobile, der sehr wohl den Anspruch erhebt, eine Repräsentation und zugleich auch eine Simulation der Vereinigten Staaten zu sein. Doch liegt dieser Band außerhalb des Radius und der Gattungszugehörigkeit der Reiseliteratur, weshalb auf ihn nicht weiter verwiesen werden soll. Denn darum geht es in einer anderen Vorlesung.

Doch kommen wir vor diesem Hintergrund zu einem letzten hier aufgegriffenen Punkt bei der Auseinandersetzung mit Baudrillards wirklich gelungenem und mehr noch denk-würdigem Reisetext. Im Grunde ist Baudrillards Amerika zugleich auch die Anleitung für eine philosophische Alterität, die eben nicht nur kulturell bedingt ist, sondern gleichsam eine außermenschliche, ja fast schon eine unmenschliche Alterität bezeichnet. Der Welt der Zeichen, der Welt der Geschichte, tritt der vom Menschen nicht letztendlich kontrollierte Raum der Wüste als inkommensurable Alterität gegenüber. Man darf sich allerdings fragen, ob sich die Weite dieses Raumes letztlich nicht doch wieder jenem amerikanischen Traum zuordnen ließe, dem sich schon die ersten Entdecker hingaben: jener unermesslichen Weite eines Landes, das mehr oder minder unberührt ist, und dessen Begehung letztlich eine Flucht aus der eigenen Geschichte und ihrer eigenen Kausalität bedeutet und zugleich verspricht. Ist denn der American Dream nicht letztlich grundlegend an 
die Oberfläche geknüpft, kurzum ein Oberflächenphänomen, das die Tiefendimensionen, welche von ihm negiert werden, unberührt und unberücksichtigt lässt?

Insoweit wäre Amérique letztlich als das Bordbuch eines neuen Columbus zu lesen, der in seinen hermeneutischen Bewegungen, in seiner Schifffahrt durch die Wüste des Meeres aus der Gefangenschaft einer Geschichte im doppelten Sinne entspringt, einer Geschichte, die freilich mit ihm und durch ihn eine neue Wendung nehmen wird. Amérique ist damit ein neuer Diario de a bordo, dem nicht die Entdeckung an sich, sondern der Weg selbst von Bedeutung wird, eine Welt, in der die Zeichen spärlich geworden sind und jeder Deutungsakt zählt. Und mehr noch: eine andere Welt als Welt des Anderen - was dem Menschen entgegensteht als Objekt, als Gegen-Stand, als Ort einer radikalen Alterität, die das Eigene durch das absolut Andere anders zu perspektivieren und neu zu ordnen erlaubt. So werden die Karten der Geschichte neu gemischt. Die Reiseliteratur von Jean Baudrillard zeigt den Ort auf, an welchem die Diskontinuität hermeneutischer Bewegungen sich auf die Abstraktion philosophischer Erörterungen öffnet, welche die Grundlagen des abendländischen Denkens zumindest in Frage stellen. Ob dies allerdings nicht Roland Barthes mit L'Empire des signes deutlich früher und wesentlich radikaler gelang, ist eine Frage, die wir zur Beantwortung an dieser Stelle unserer Vorlesung ganz bewusst offen lassen. 